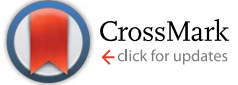

Cite this: RSC AdV., 2017, 7, 11821

Received 18th November 2016 Accepted 10th February 2017

DOI: 10.1039/c6ra26991e

rsc.li/rsc-advances

\section{Aptamer functionalized silver clusters for STED microscopy}

\author{
Lan Wang, ${ }^{a}$ Haisen $\mathrm{Ta}^{\mathrm{b}}{ }^{\mathrm{C}}$ Chaitanya Ullal, ${ }^{\mathrm{c}}$ Fu Wang, ${ }^{\text {*a }}$ Chuanyi Wang ${ }^{\mathrm{a}}$ \\ and Guohui Dong ${ }^{a}$
}

Alternative to traditional dye or fluorescent protein, a novel stable probe for stimulated emission depletion (STED) microscopy is critically needed. In this work, aptamer functionalized Ag clusters with high photostability were successfully prepared. Moreover, improved resolution has been observed both at the single particle level and in specific cell structures, suggesting that aptamer functionalized Ag clusters are promising candidates for STED microscopy.

\section{Introduction}

For more than a century, the spatial resolution of light microscopy was thought to be fundamentally limited, as described by Abbé's theory, which states that the resolution cannot be better than half the wavelength of the applied light. ${ }^{1-3}$ When imaging with visible light, the resolution is typically limited to $\sim 200 \mathrm{~nm}$ in the lateral direction and $\sim 500 \mathrm{~nm}$ in axial directions, which inhibits high-resolution imaging of subcellular structure and hinders a molecular-level understanding of cell structure and dynamics. ${ }^{4}$ Stimulated emission depletion (STED) microscopy was the first concept that defeated the diffraction barrier by shrinking the effective point spread function (PSF), ${ }^{5,6}$ fluorescence emission in the periphery of the diffraction limited focal spot is depleted by additional laser light, whose wavelength is tuned to the red edge of the emission spectrum of the fluorophore. The resolution of STED microscopy, depends primarily on the intensity of the depletion laser, and has no theoretical limitation. ${ }^{3}$ Recent studies unveiling cellular details with resolution better than $100 \mathrm{~nm}$ have been achieved in cells, living tissue and organisms. ${ }^{7-10}$ Locating targets of interest requires delivery of appropriate fluorophores in close proximity of the target structure. Typically, a pair of primary and dye labelled secondary antibodies is used for fluorescent staining. Since the size of a common antibody is on the order of $10 \mathrm{~nm}$, therefore the actual distance between the protein of interest and the observed fluorescent spot can be as large as $20 \mathrm{~nm} .^{7,11}$ Moreover, due to spatial constraints, the

${ }^{a}$ Laboratory of Environmental Sciences and Technology, Key Laboratory of Functional Materials and Devices for Special Environments, Xinjiang Technical Institute of Physics \& Chemistry, Chinese Academy of Sciences, Urumqi 830011, China. E-mail: wangfu@ ms.xjb.ac.cn; Fax: +86-991-3838957; Tel: +86-991-3835879

${ }^{b}$ Department of NanoBiophotonics, Max Planck Institute for Biophysical Chemistry, Göttingen 37077, Germany

${ }^{c}$ Department of Materials Science and Engineering, Rensselaer Polytechnic Institute, Troy, New York 12180, USA antibody based labelling strategy cannot achieve the level of binding to every target molecule, resulting in spotty images. ${ }^{12-14}$ Alternatives to antibody staining, such as genetically fused fluorescent proteins and tag-based protein labelling strategies have been established for STED microscopy. ${ }^{15,16}$ However, the introduction and overexpression of foreign coding in a cell might lead to undesired protein aggregation, mis-localization, and loss or gain of function of the target. ${ }^{17,18}$

Aptamer based labelling has been proposed as a complement to the use of existing probes as they possess many favourable characteristics in terms of low molecular weight, little or no immune response, low cost, and ease of preparation and modification. ${ }^{19-22}$ Aptamers are single-stranded DNA or RNA oligonucleotides with molecular weights of 10-15 kDa and lengths down to $2-3 \mathrm{~nm}$, which can spontaneously fold into well-defined 3-dimensional structures and bind to a broad diversity of targets with high affinity and specificity, such as proteins, phospholipids, sugars, nucleic acids, and even whole bacteria and cells. ${ }^{20-24}$ In 2012, Opazo et al. demonstrated the potential of dye coupled aptamers for super-resolution imaging by imaging details of endosomal structures such as thin endosomal tubules. ${ }^{25}$ However, the photostability of traditional fluorescent dyes remains a concern for super resolution microscopy. In contrast, oligonucleotide stabilized fluorescent silver clusters have emerged as an alternative class of fluorescence contrast agents with distinct advantages.$^{26-28}$ Fluorescent silver clusters, featured by their subnanometer size, good quantum yield and photostability, low toxicity, high extinction coefficients, large Stokes shift, tuneable emission spectrum, and unobtrusive blinking on all relevant time scales, fill a void between organic dye and much larger semiconductor-based probes. $^{28-31}$

In this paper, we report on the preparation of aptamer functionalized silver clusters and their viability of using aptamer functionalized silver clusters as fluorophores for STED microscopy. Specifically, the aptamer functionalized silver 
clusters were constructed by linking the anti-proliferative G-rich aptamer AS1411 with a single stranded CCCTTAATCCC DNA oligomer. The aptamer AS1411 was used to specifically bind with the nucleolin protein in the plasma membranes of cancer cells, ${ }^{32,33}$ while the selected CCCTTAATCCC worked as the template for the formation of the silver cluster. The as prepared silver clusters have a fluorescence quantum yield of $35 \%$, and show maximum absorption and emission at $590 \mathrm{~nm}$ and $640 \mathrm{~nm}$, respectively. To STED microscopy, the benefit of the asprepared silver clusters is to be effectively de-excited by $770 \mathrm{~nm}$ laser. Improved stability was observed compared to Alexa Fluor 594 and Atto 594. The specificity of aptamer functionalized silver clusters was evaluated using confocal microscopy by comparing the performance between a cancer cell line (Hela) and a normal cell line (Chinese hamster ovary, $\mathrm{CHO}$ ), the results clearly showing that the AS1411 functionalized silver clusters can bind to the Hela cells. Nucleolin protein distribution in Hela cells was demonstrated in STED microscopy with resolved resolution, proving that the aptamer functionalized $\mathrm{Ag}$ cluster is a promising fluorescent probe for STED microscopy.

\section{Experimental}

\section{Chemicals and materials}

Silver nitrate (99.99\%), sodium borohydride, $\mathrm{NaBH}_{4}$ (powder, 98\%), disodium hydrogen phosphate, sodium dihydrogen phosphate, trisodium citrate, citric acid, paraformaldehyde, 4,6-diamidino-2-phenylindole (DAPI), rhodamine 6G, ATTO 594 and cell cultures were all purchased from SIGMA ALDRICH. Alexa Fluor 594 was purchased from Thermo Fisher Scientific. All single strand DNA were synthesized and purified by EBA Co. Ltd. (Germany). All other chemicals involved in this work were analytical-grade. All aqueous solutions were freshly prepared in ultrapure water ( $\geq 18 \mathrm{M} \Omega$, Milli-Q, Millipore).

\section{Synthesis of fluorescent silver nanoclusters}

DNA stabilized AgNCs were synthesized according to previous procedure with slight modification. ${ }^{32}$ In brief, $3 \mu \mathrm{M}$ DNA template and $20 \mu \mathrm{M} \mathrm{AgNO}{ }_{3}$ were sequentially added and mixed with various buffer by vigorous shaking for $1 \mathrm{~min}$ in an Eppendorf tube, and then kept at room temperature in the dark. After $20 \mathrm{~min}, 20 \mu \mathrm{M}$ freshly prepared $\mathrm{NaBH}_{4}$ was added, thoroughly mixed, and then stored without further mixing at room temperature in the dark for 12 hours. The ratio of base to $\mathrm{Ag}^{+}$ and to $\mathrm{BH}_{4}{ }^{-}$was $2: 1: 1$.

\section{Apparatus and characterization}

UV-vis spectra were recorded on a UV-3600 spectrophotometer, and fluorescence spectra were recorded using an F-4500 fluorescence spectrophotometer. TEM images were recorded on a JEM-2100F operated at $200 \mathrm{kV}$. The fluorescence lifetime and STED imaging were test on home build super-resolution microscopy. Other whole cell imaging and nuclear staining study were conducted with a commercial confocal microscope (TCS SP5, Leica, Germany).

\section{Quantum yield measurement}

PL quantum yields (QYs) were determined by comparing the integrated emission of the samples in water with that of rhodamine 6G (QY $=94 \%$ in ethanol) or fluorescent Atto 594 $(\mathrm{QY}=85 \%$ in water) according to the following equation:

$$
Q=Q_{\mathrm{R}} \frac{I}{R_{\mathrm{R}}} \frac{\mathrm{OD}_{\mathrm{R}}}{\mathrm{OD}} \frac{n^{2}}{n_{\mathrm{R}}^{2}}
$$

where $Q$ is the quantum yield, $I$ is the measured integrated emission intensity, $n$ is the refractive index, and OD is the optical density. The subscript $\mathrm{R}$ refers to the reference fluorophore of known quantum yield. In order to minimize reabsorption effects absorbance in the $10 \mathrm{~mm}$ fluorescence cuvette were kept under 0.05 at the excitation wavelength.

\section{STED and confocal imaging}

Single color STED microscopy was performed as described. ${ }^{34,35}$ STED and the corresponding confocal images were recorded by piezo stage scanning equipped with a pulsed excitation laser at 595 nm (PicoQuant, Berlin, Germany) and a Titanium-Sapphire STED laser emitting $770 \mathrm{~nm}$ pulses with reputation rate of 80 MHz. The STED beam passes a polymeric phase plate (Vortex pattern, RPC Photonics, NY, USA). In combination with a $\lambda / 4$ waveplate, the phase plate is responsible for the doughnut shaped STED-focus. The excitation and the STED beams were co-aligned and coupled into a 1.4 numerical aperture oil immersion lens (NA 1.4 HCX PL APO, 100x, Leica Microsystems, Wetzlar, Germany). The fluorescence was collected by the same lens, spectrally separated from 620 to $680 \mathrm{~nm}$. The fluorescence was detected by fiber-coupled single-photon-counting modules (SPCMAQRH13, Perkin Elmer), with their fiber cores acting as confocal pinholes.

\section{Preparation of samples for confocal and STED microscopy}

Immobilized single-molecule experiments were performed by spun-coating diluted Alexa Fluor 594, Atto 594 and fluorescent $\mathrm{Ag}$ cluster solution on a poly-L-lysine coated glass coverslip at $1500 \mathrm{rpm}$ for $1 \mathrm{~min}$. Coverslips were cleaned by sequential sonication in acetone, $1 \mathrm{M} \mathrm{NaOH}$, and Milli-Q water, each for 15 min. The coverslips were then rinsed with Milli-Q water, dried with compressed $\mathrm{N}_{2}$.

The cultivation of cells, and immune labelling was based on established protocols. ${ }^{32,36}$ HeLa cells $\left(1 \times 10^{5}\right)$ were seeded onto $12 \mathrm{~mm}$ sterile coverslips in a 24-well plate. After $24 \mathrm{~h}$, cells were incubated with AS1411-A5-AgNCs for $30 \mathrm{~min}$ at $4{ }^{\circ} \mathrm{C}$ after being washed three times with phosphate-buffered saline (PBS). To remove the unbound AgNCs, the cells were washed three times with PBS. Then cells were fixed for $20 \mathrm{~min}$ in $200 \mu \mathrm{L}$ of $3.7 \%$ paraformaldehyde. After the fixation, cells were washed three times with PBS, followed by nucleus staining with DAPI. Ten minutes later, the cells were further washed three times with PBS buffer. The side of coverslip with fixed cells were mounted in Mowiol. In addition, HeLa cell with CCCTTAATCCC functionalized Ag cluster and CHO cell with AS1411-A5-AgNCs were also prepared as control samples. 


\section{Results and discussion}

Ag clusters, termed as AgNCs, were prepared by simply mixing single strand CCCTTAATCCC DNA scaffold and silver nitrate in a phosphate buffer, followed by reduction with sodium borohydride. As shown in Fig. 1, the type of buffer, $\mathrm{pH}$ value and concentration of the buffer play important roles in the synthesis of luminescent Ag clusters, which might be due to the folding structure of aptamers being slightly different in various conditions. ${ }^{32,37}$ Four different buffers were compared in the synthesis of AgNCs, as shown in Fig. 1A, phosphate buffer gives the highest fluorescence with maximum emission around $560 \mathrm{~nm}$ when excited at $470 \mathrm{~nm}$. The $\mathrm{pH}$ value and concentration of phosphate buffer (Fig. 1B and C) demonstrated that $20 \mathrm{mM}$ phosphate buffer with $\mathrm{pH}$ at 7.0 present the strongest fluorescence emission. These optimized parameters were also used in the synthesis of aptamer functionalized AgNCs.

The resultant AgNCs have an excitation maximum of $\sim 470 \mathrm{~nm}$ and an emission maximum $\sim 560 \mathrm{~nm}$ (Fig. 2A). The corresponding fluorescence quantum yield determined by comparison with rhodamine $6 \mathrm{G}$ was $\sim 17 \%$, and the AgNCs exhibit a fluorescence lifetime of 2.32 ns with a mono-exponential behavior (Fig. 2B), suggesting monodispersity of the AgNCs.

Aptamer functionalized AgNCs were prepared under the same conditions, with the exception of using AS1411 linked CCCTTAATCCC template. To avoid the incidence of steric hindrance, ${ }^{38}$ an AAAAA spacer was found more bright (termed as AS1411-A5-AgNCs) than the previously used TTTTT spacer. ${ }^{32}$ The resulted AS1411-A5-AgNCs show strong red emission with quantum yield of 35\% using ATTO 594 as the reference standard. As shown in Fig. 3A, with a maximum excitation at around $570 \mathrm{~nm}$, the emission peak is about $650 \mathrm{~nm}$, the value is slightly red shift in excitation wavelength between $550 \mathrm{~nm}$ and $610 \mathrm{~nm}$ (Fig. 3C). The lifetime of AS1411-A5-AgNCs is 2.47 ns (Fig. 3B). It is well approximated by a single exponential decay, indicating that a single species dominates the emission. The red shift in the emission spectra and increased lifetime of the AgNCs suggest that the added DNA sequence influences the property of the final AgNCs. A similar effect was observed on aptamer functionalized single stranded DNA consisting of 12 cytosines. ${ }^{32}$

To confirm that CCCTTAATCCC template is critical to the formation of the AgNCs, aptamer mucin 1 was incorporated onto CCCTTAATCCC using an AAAAA spacer, termed as mucin-A5AgNCs. The resulted excitation and emission peaks are located at $590 \mathrm{~nm}$ and $650 \mathrm{~nm}$ (Fig. 4A), respectively. The fluorescent decay profiles (Fig. 4B) once again display a mono-exponential fit
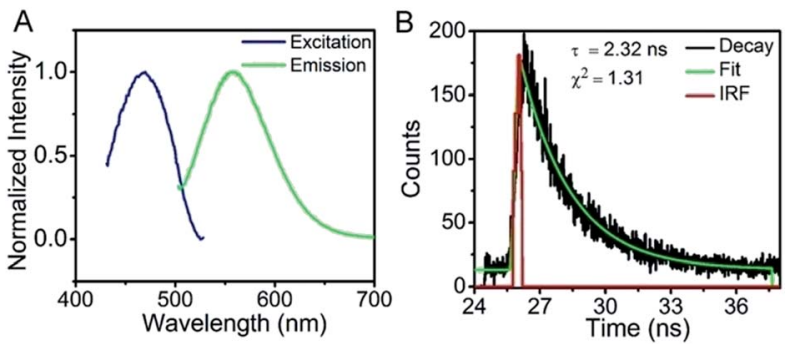

Fig. 2 (A) Excitation and emission spectra of AgNCs, (B) decay lifetime of AgNCs excited at $488 \mathrm{~nm}$, black line is measured data, green curve represent fits, where red IRF represent instrument response function.

with a lifetime of $2.54 \mathrm{~ns}$. It should be mentioned that in the absence of CCCTTAATCCC, both AS1411 and mucin 1 do not produce significant fluorescence, suggesting that CCCTTAATCCC is crucial for the formation of fluorescent silver clusters, while the aptamer mainly serves the role of labelling the target structure and only slightly influences the spectra.

Size and elemental mapping of the AS1411-A5-AgNCs were performed using high-angle annular dark field (HAADF) imaging and EDX analysis. It can be seen in Fig. 5 that the

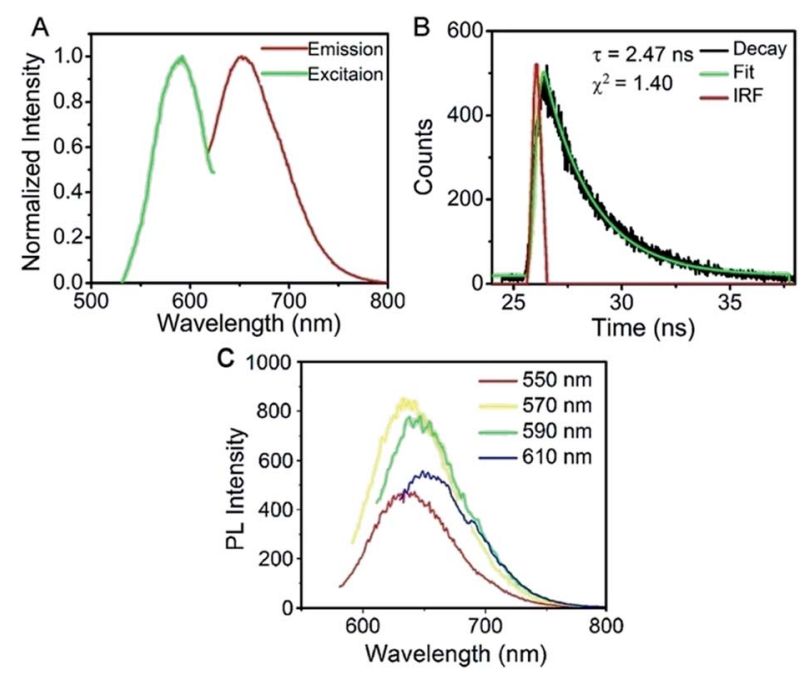

Fig. 3 (A) Excitation spectra and fluorescence emission spectra of AS1411-A5-AgNCs. (B) Fluorescence lifetime of AS1411-A5-AgNCs dispersed in phosphate buffer, excitation wavelength: $590 \mathrm{~nm}$. (C) Emission spectra of AS1411-A5-AgNCs when excited from $550 \mathrm{~nm}$ to $610 \mathrm{~nm}$.
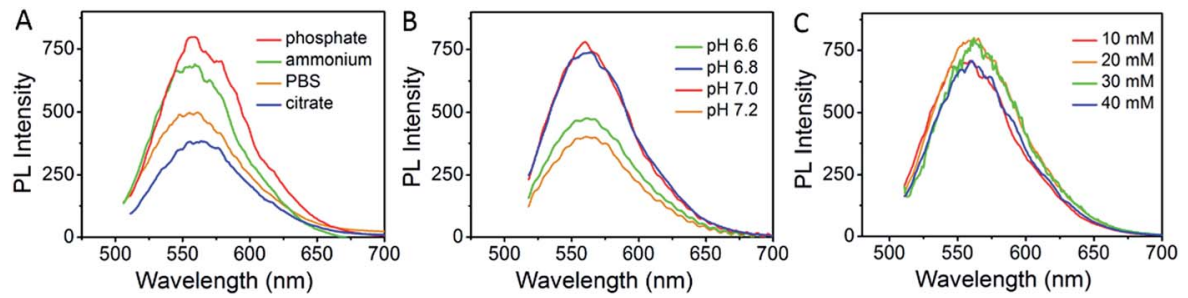

Fig. 1 Optimizing the synthesis of AgNCs. (A) Buffer selection, (B) pH value and (C) concentration of phosphate buffer selection. 

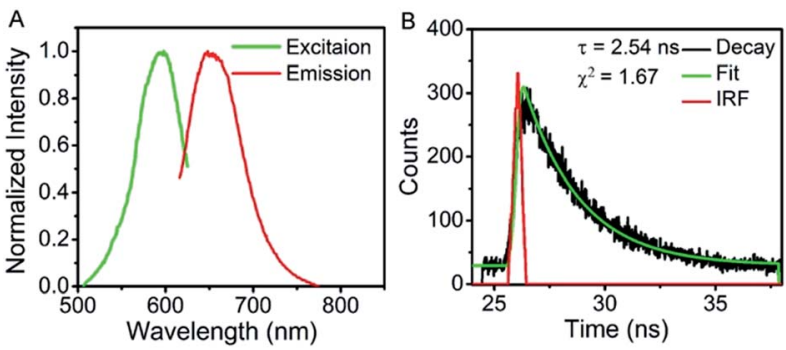

Fig. 4 (A) Excitation and emission spectra of mucin-A5-AgNCs, (B) decay lifetime of mucin-A5-AgNCs excited at $595 \mathrm{~nm}$, black line is measured data, green curve represent fits, where red IRF represent instrument response function.

AS1411-A5-AgNCs are monodispersed with a size distribution around $1.8 \mathrm{~nm}$, while the EDX pattern confirms that the major constituent of clusters is Ag. The major elements like the $\mathrm{Na}, \mathrm{C}$, $\mathrm{O}, \mathrm{P}, \mathrm{Cu}$ should came from the buffer and the TEM substrate.

A stage scanning STED microscope was built to evaluate the performance of single AS1411-A5-AgNCs (Fig. 6A). A picosecond $595 \mathrm{~nm}$ laser with $80 \mathrm{MHz}$ pulse train was used for excitation (yellow), while a $770 \mathrm{~nm}$ mode-locked Ti:sapphire laser whose output was engineered to produce a donut shaped focal spot (red) was used to switch off peripheral fluorescence, the shrunk fluorescence was collected by a detector (orange). As shown in Fig. 6B, isolated bright point-like fluorescent objects (around $220 \mathrm{~nm}$ ), most likely due to individual AS1411-A5-AgNCs clusters, were observed in confocal image. In contrast, the STED counterpart (Fig. 6C) shows excellent resolution improvement with good signal-to noise ratio, especially the objects marked in white cycles, which are difficult to distinguish in confocal image, but well separated in STED image. For a power of $70 \mathrm{~mW}$ in the focal plane, an average optical resolution of approximately $52 \mathrm{~nm}$ was achieved. It should be noted that this resolution is attained in raw data, exclusively based on optically induced molecular transitions. Further increasing the power of STED laser should lead to an improvement in resolution. However, the high power depletion light also increases the probability to drive the AS1411-A5-AgNCs transiting into a recoverable long-lived dark state, which is similar to previous observation..$^{39,40}$ For instance, upon increasing the STED power to $120 \mathrm{~mW}$, almost one fourth of the clusters go to the dark
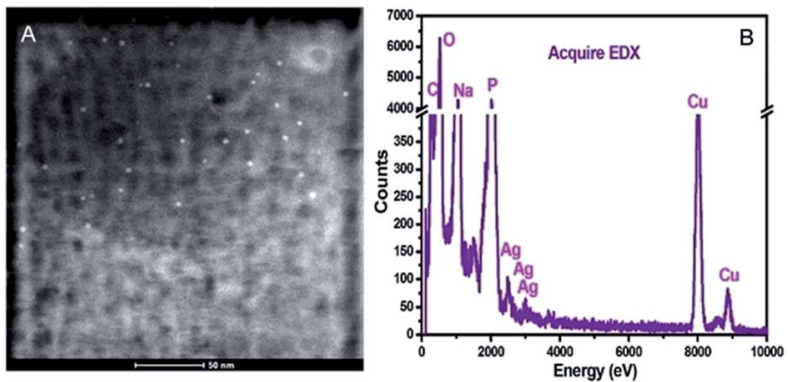

Fig. 5 High-angle annular dark field (HAADF) image (A) and EDX analysis (B) of AS1411-A5-AgNCs.
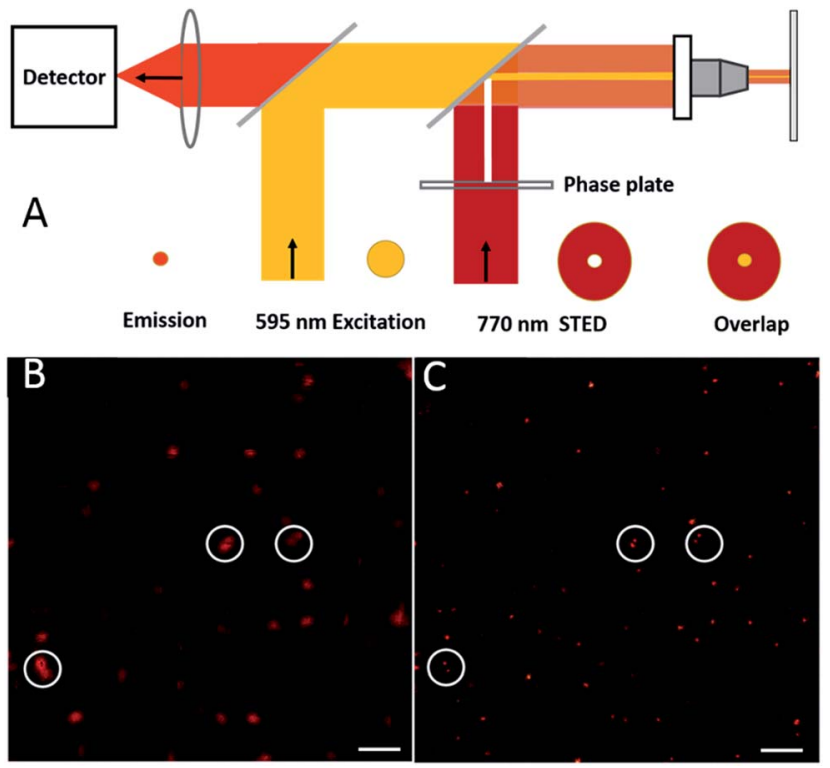

Fig. 6 (A) Schematic diagram of an STED microscope with a doughnut shaped STED beam (red), overlapped with the excitation beam (yellow), and the resulting effective fluorescence emission (orange). (B) Confocal and (C) STED images of AS1411-A5-AgNCs on coverslip. Scale bar: $1 \mu \mathrm{m}$

state, before gradually turning fluorescent over the timescale of seconds. Previously research demonstrated that charge transfer from $\mathrm{Ag}$ to DNA mechanism possibly give rise to the photo induce dark states. ${ }^{41}$ In order to further improving the performance of the Ag clusters as what have been done to reduce the blinking of quantum dots, ${ }^{42}$ more extensive evaluation of the dark state need to be pursued in future studies.

The photostability of AS1411-A5-AgNCs under confocal and STED conditions was evaluated, quantified and compared with two other conventional STED probes, Alexa flour 594 and ATTO 594. For this purpose, thin layers of AS1411-A5-AgNCs and dyes were spin-coated on poly-L-lysine coated glass coverslips. The normalized total fluorescence signal from a $2 \times 2 \mu \mathrm{m}$ size area (averaging from 3 different selected areas) in the samples over the course of 20 scans were then compared. Excitation and
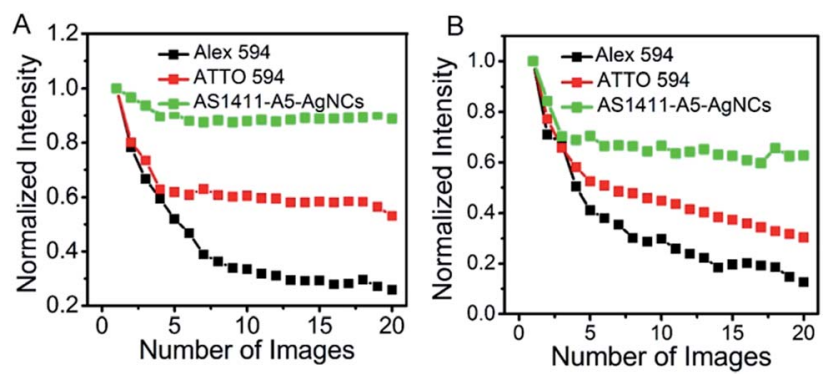

Fig. 7 Photostability of AS1411-A5-AgNCs and the commercial dyes ATTO 594 and Alexa 594. Bleaching curves were measured by taking consecutive (A) confocal and (B) STED microscopy images of the samples spun coated onto coverslips. The excitation and STED powers are $10 \mathrm{~W}$ and $100 \mathrm{~mW}$, respectively. 


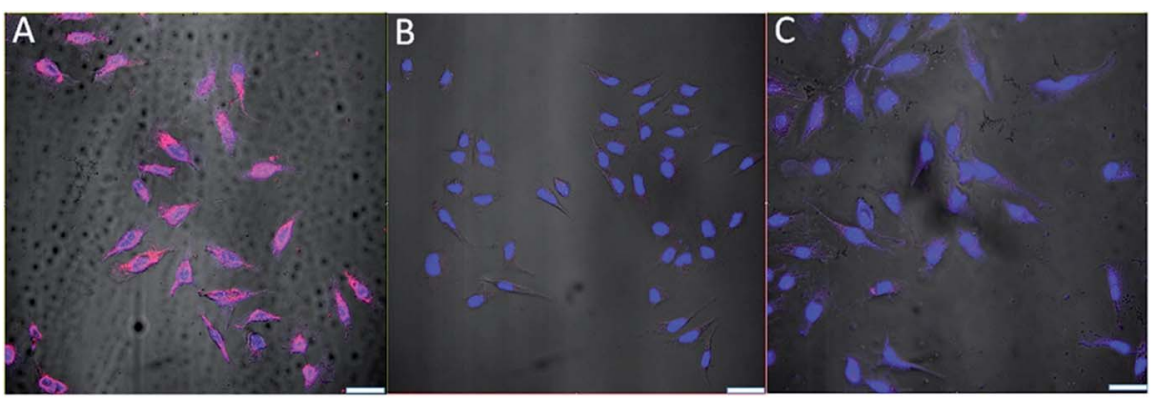

Fig. 8 Overlapped confocal and bright field images of AS1411-A5-AgNCs incubated with (A) cancer HeLa and (B) healthy CHO cells. (C) AgNCs incubated with cancer HeLa cells. The blue color is the DAPI stained nucleus, while magenta indicates the nucleolin in the plasma membranes of cancer cells. The AS1411-A5-AgNCs and AgNCs were excited with $543 \mathrm{~nm}$. Scale bar: $20 \mu \mathrm{m}$.

STED powers at the objective back aperture of $10 \mathrm{~mW}$ and 100 $\mathrm{mW}$ were used, respectively. As shown in Fig. 7, the AS1411-A5AgNCs exhibit superior photostability compared with Alexa flour 594 and ATTO 594 under both confocal and STED conditions. The fluorescence of AS1411-A5-AgNCs persists after a relatively fast drop in the first few scans then to $\sim 90 \%$ and $\sim 60 \%$ after 20 scans in confocal and STED, respectively. In contrast, the fluorescence of Alexa flour 594 dramatically decreased to $26 \%$ and $13 \%$, and ATTO 594 decreased to $53 \%$ and $30 \%$, respectively.

The aptamer AS1411 is reported to bind to nucleolin in the plasma membranes of cancer cells. ${ }^{32,33}$ To verify if the AS1411A5-AgNCs could be used as a specific fluorescent probe for cancer cell imaging, the sample was incubated with HeLa and healthy $\mathrm{CHO}$ cells for $30 \mathrm{~min}$ at $4{ }^{\circ} \mathrm{C}$ and subsequently washed with PBS buffer. To increase phase-contrast, the nucleus of both cell lines were stained with DAPI (blue). As illustrated in Fig. 8, strong fluorescence (magenta) along the cellular membranes was observed in the HeLa cells (Fig. 8A), while no significant fluorescence signal was recorded for the $\mathrm{CHO}$ cells (Fig. 8B). The confocal microscopy images confirm that AS1411-A5-AgNCs bind specifically to nucleolin protein in the cellular membrane of cancer cells. Additional control experiment was performed by replacing AS1411-A5-AgNCs with AgNCs during incubation with HeLa cells; comparatively negligible fluorescence was observed from the plasma membranes in the corresponding image (Fig. 8C). Therefore, AS1411-A5-AgNCs preserve the specificity of the aptamer.

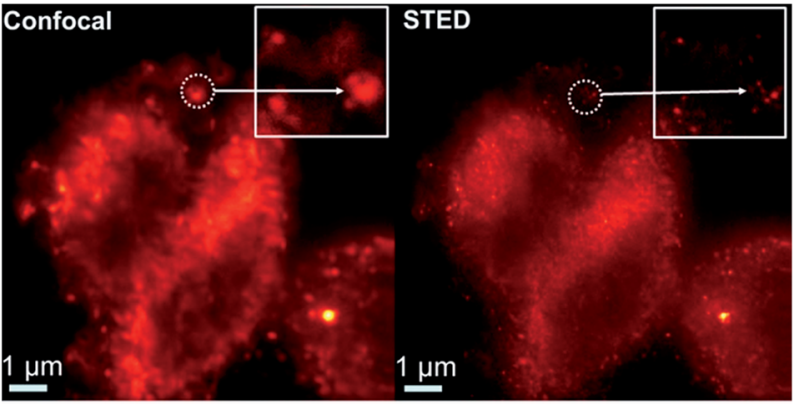

Fig. 9 Confocal and STED images of AS1411-A5-AgNCs labeled Hela cells. STED power is $80 \mathrm{~mW}$.
A closer observation of the distribution of AS1411-A5-AgNC labelled nucleolin in plasma membranes was further performed under confocal and STED conditions (Fig. 9). From the confocal image, it is hard to resolve the detail of the structure, while their STED counterpart consistently provide superior contrast and resolution. The specificity and reliability of the AS1411-A5-AgNC in compare with the conventional antibody labelling strategy is vital to promote its practical application and worthy to perform further.

\section{Conclusions}

In summary, aptamer functionalized fluorescent $\mathrm{Ag}$ clusters were successfully synthesized by integrating AS1411 to a new DNA scaffold via an AAAAA loop. The maximum absorption and emission of the prepared AS1411-A5-AgNCs are located at $590 \mathrm{~nm}$ and $640 \mathrm{~nm}$, and the fluorescence quantum yield is $35 \%$. The AS1411-A5-AgNCs demonstrate to have specific binding affinity to nucleolin in the plasma membranes of Hela cells. Moreover, AS1411-A5-AgNCs are proved to be an excellent probe for STED microscopy both at the single cluster level and in cell imaging. Considering the biological and chemical advantages of aptamers over antibodies and fluorescent proteins, including the small size, ease of production and chemical modification, and high affinity to targets, in combination with the photostability of silver clusters, the proposed aptamer functionalized silver clusters offer a promising labelling strategy for novel analytical and biomedical applications and will open up a new avenue for synthesizing small but stable fluorescent probes for super resolution microscopy.

\section{Acknowledgements}

Financial support by the '1000 Talent Program' (The Recruitment Program of Global Experts), and the National Natural Science Foundation of China $(21503271,21303258)$.

\section{References}

1 S. W. Hell, Angew. Chem., Int. Ed., 2015, 54, 8054-8066.

2 E. Abbe, Archiv für mikroskopische Anatomie, 1873, 9, 413-418.

3 S. W. Hell, Nat. Methods, 2009, 6, 24-32. 
4 P. Hunter, EMBO Rep., 2011, 12, 643-646.

5 S. W. Hell, Nat. Biotechnol., 2003, 21, 1347-1355.

6 T. A. Klar, S. Jakobs, M. Dyba, A. Egner and S. W. Hell, Proc. Natl. Acad. Sci. U. S. A., 2000, 97, 8206-8210.

7 H. Blom and H. Brismar, J. Intern. Med., 2014, 276, 560-578.

8 R. Han, Z. Li, Y. Fan and Y. Jiang, J. Genet. Genomics, 2013, 40, 583-595.

9 R. Chéreau, J. Tønnesen and U. V. Nägerl, Methods, 2015, 88, 57-66.

10 T. Müller, C. Schumann and A. Kraegeloh, ChemPhysChem, 2012, 13, 1986-2000.

11 G. Patterson, M. Davidson, S. Manley and J. LippincottSchwartz, Annu. Rev. Phys. Chem., 2010, 61, 345-367.

12 T. Lang and S. O. Rizzoli, Physiology, 2010, 25, 116-124.

13 U. Agrawal, D. T. Reilly and C. M. Schroeder, Curr. Opin. Biotechnol., 2013, 24, 646-653.

14 F. Opazo, Super-Resolution Microscopy Techniques in the Neurosciences, 2014, vol. 86, pp. 369-386.

15 F. Stagge, G. Y. Mitronova, V. N. Belov, C. A. Wurm and S. Jakobs, PLoS One, 2013, 8, e78745.

16 J. Chojnacki, T. Staudt, B. Glass, P. Bingen, J. Engelhardt, M. Anders, J. Schneider, B. Muller, S. W. Hell and H. G. Krausslich, Science, 2012, 338, 524-528.

17 D. M. Chudakov, M. V. Matz, S. Lukyanov and K. A. Lukyanov, Physiol. Rev., 2010, 90, 1103-1163.

18 M. W. Davidson and R. E. Campbell, Nat. Methods, 2009, 6, 713-717.

19 A. Aravind, Y. Yoshida, T. Maekawa and D. S. Kumar, Drug Delivery Transl. Res., 2012, 2, 418-436.

20 S. D. Jayasena, Clin. Chem., 1999, 45, 1628-1650.

21 K. C. Liu, B. S. Lin and X. P. Lan, J. Cell. Biochem., 2013, 114, 250-255.

22 A. Z. Wang and O. C. Farokhzad, J. Nucl. Med., 2014, 55, 353356.

23 C. L. A. Hamula, H. Q. Zhang, L. L. Guan, X. F. Li and X. C. Le, Anal. Chem., 2008, 80, 7812-7819.

24 C. X. Zhang, X. F. Lv, X. Han, Y. Man, Y. Saeed, H. Qing and Y. L. Deng, Anal. Methods, 2015, 7, 6339-6345.

25 F. Opazo, M. Levy, M. Byrom, C. Schafer, C. Geisler, T. W. Groemer, A. D. Ellington and S. O. Rizzoli, Nat. Methods, 2012, 9, 938-939.
26 J. T. Petty, J. Zheng, N. V. Hud and R. M. Dickson, J. Am. Chem. Soc., 2004, 126, 5207-5212.

27 B. Y. Han and E. K. Wang, Anal. Bioanal. Chem., 2012, 402, 129-138.

28 S. Choi, R. M. Dickson and J. H. Yu, Chem. Soc. Rev., 2012, 41, 1867-1891.

29 X. H. Tan and R. C. Jin, Wiley Interdiscip. Rev.: Nanomed. Nanobiotechnol., 2013, 5, 569-581.

30 L. Shang, S. J. Dong and G. U. Nienhaus, Nano Today, 2011, 6, 401-418.

31 Y. Z. Lu and W. Chen, Chem. Soc. Rev., 2012, 41, 3594-3623.

32 J. L. Li, X. Q. Zhong, F. F. Cheng, J. R. Zhang, L. P. Jiang and J. J. Zhu, Anal. Chem., 2012, 84, 4140-4146.

33 H. G. Sun, X. Zhu, P. Y. Lu, R. R. Rosato, W. Tan and Y. L. Zu, Mol. Ther.-Nucleic Acids, 2014, 3, e182.

34 F. Göttfert, C. A. Wurm, V. Mueller, S. Berning, V. C. Cordes, A. Honigmann and S. W. Hell, Biophys. J., 2013, 105, L01L03.

35 C. A. Wurm, K. Kolmakov, F. Göttfert, H. Ta, M. Bossi, H. Schill, S. Berning, S. Jakobs, G. Donnert, V. N. Belov and S. W. Hell, Opt. Nanoscopy, 2012, 1, 1-7.

36 J. B. Zhu, L. B. Zhang, Y. Teng, B. H. Lou, X. F. Jia, X. X. Gu and E. K. Wang, Nanoscale, 2015, 7, 13224-13229.

37 C. I. Richards, S. Choi, J. C. Hsiang, Y. Antoku, T. Vosch, A. Bongiorno, Y. L. Tzeng and R. M. Dickson, J. Am. Chem. Soc., 2008, 130, 5038-5039.

38 A. Latorre, R. Lorca and A. Somoza, J. Chem., 2013, 631421.

39 J. Sharma, H. C. Yeh, H. Yoo, J. H. Werner and J. S. Martinez, Chem. Commun., 2010, 46, 3280-3282.

40 T. Vosch, Y. Antoku, J. C. Hsiang, C. I. Richards, J. I. Gonzalez and R. M. Dickson, Proc. Natl. Acad. Sci. U. S. A., 2007, 104, 12616-12621.

41 S. A. Patel, M. Cozzuol, J. M. Hales, C. I. Richards, M. Sartin, J. C. Hsiang, T. Vosch, J. W. Perry and R. M. Dickson, J. Phys. Chem. C, 2009, 113, 20264-20270.

42 X. Y. Wang, X. F. Ren, K. Kahen, M. A. Hahn, M. Rajeswaran, S. Maccagnano-Zacher, J. Silcox, G. E. Cragg, A. L. Efros and T. D. Krauss, Nature, 2009, 459, 686-689. 